\title{
ERROR-RELATED POTENTIAL RECORDED BY EEG IN THE CONTEXT OF A P300 MIND SPELLER BRAIN-COMPUTER INTERFACE
}

\author{
Adrien Combaz ${ }^{1}$, Nikolay Chumerin ${ }^{1}$, Nikolay V. Manyakov ${ }^{1}$, Arne Robben ${ }^{1}$, Johan A. K. Suykens ${ }^{2}$ and \\ Marc M. Van Hulle
}

${ }^{1}$ K.U.Leuven, Laboratorium voor Neuro- en Psychofysiologie, Herestraat 49, B-3000 Leuven, Belgium

${ }^{2}$ K.U.Leuven, ESAT-SCD, Kasteelpark Arenberg 10, BE-3001 Heverlee, Belgium

\begin{abstract}
The Mind Speller ${ }^{\circledR}$ is a Brain-Computer Interface (BCI) which enables subjects to spell text on a computer screen by detecting P300 Event-Related Potentials in their electroencephalograms (EEG). This BCI application is of particular interest for disabled patients who have lost all means of verbal and motor communication. Error-related Potentials (ErrP) in the EEG are generated by the subject's perception of an error. We report on the possibility of using this ErrP for improving the performance of our Mind Speller ${ }^{\circledR}$. We tested 6 subjects and recorded several typing sessions for each of them. Responses to correct and incorrect performances of the BCI are recorded and compared. The shape of the received ErrP is compared to other studies. The detection of this ErrP and its integration in the Mind Speller ${ }^{\circledR}$ are discussed.
\end{abstract}

\section{INTRODUCTION}

Brain Computer Interfaces (BCIs) are aimed at creating a direct communication pathway between the brain and an external device, bypassing the need for an embodiment. In the last few years, research in the field of BCI has witnessed a spectacular development (see [1], [2]) and is nowadays regarded as one of the most successful applications of the neurosciences. Indeed, such systems can provide a significant improvement of the quality of life of neurologically impaired patients suffering of pathologies such as amyotrophic lateral sclerosis, brain stroke, brain/spinal cord injury, etc...

$\mathrm{AC}$ is supported by a specialization grant from the Agentschap voor Innovatie door Wetenschap en Technologie (IWT, Flemish Agency for Innovation through Science and Technology). NC is supported by IST-2007-217077. NVM is supported by IST-2004027017. JAKS acknowledges support of FWO G.0588.09, G.0302.07, CoE EF/05/006, GOA-MANET, IUAP DYSCO. MVH is supported by EF 2005, CREA/07/027, G.0234.04, G.0588.09, IUAP P5/04, GOA 10/019, IST-2004-027017 and IST-2007217077). The authors wish to thank Refet Firat Yazicioglu, Tom Torfs and Chris Van Hoof from the Interuniversity Microelectronics Centre (IMEC) in Leuven for providing us with the wireless EEG system.

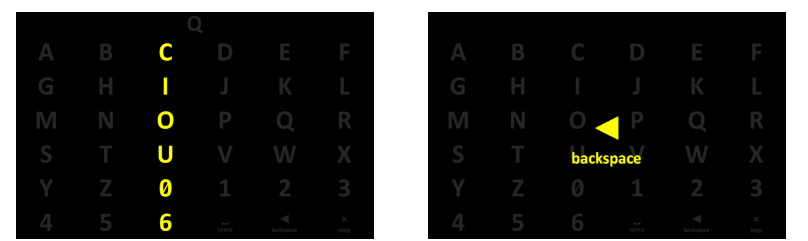

Fig. 1. User display for the P300 Mind-speller BCI. Left: intensification of a column of the matrix display. Right: Feedback moment: the identified symbol is displayed on the screen.

In invasive BCIs, a micro-electrode array is implanted in the brain (mainly in the motor or premotor frontal areas or into the parietal cortex [3]), while in non-invasive BCIs, mostly electroencephalograms (EEGs) are recorded from the scalp. There are several types of EEG-based BCIs; for example some are based on Steady State Visually Evoked Potential (SSVEP, [4]); they work by detecting the activity of the brain at a specific frequency corresponding to the flickering frequency of a visual stimulus (see [5], [6] for applications). Another type of BCIs relies on the detection of mental tasks (imagination of right/left hand movements, subtraction, word association, etc...) which are detected through slow cortical potentials (SCP) [7], readiness potential [8] and event-related desynchronization (ERD) [9].

The BCI presented here belongs to another category; it is based on the detection of the P300 Event-Related Potential (ERP: stereotyped electrophysiological response to an internal or external stimulus, [10]). This brain potential is elicited in the context of an oddball paradigm: when a subject performs the classification of two types of events, one of which is only rarely presented, the rare event will elicit in the EEG an ERP with an enhanced positive-going component at a latency of about $300 \mathrm{~ms}$ (the P300 ERP, [11]). The first spelling system based on the detection of the P300 was introduced in 1988 by Farwell and Donchin [12]. This application is nowadays one of the most studied BCI and the work presented here treats of this specific system. The Mind Speller ${ }^{\circledR}$ allows subjects to spell words by focusing on the desired characters shown in a matrix display while the rows and columns of the matrix are consecutively and randomly intensified (Fig.1-left). The intensification of a row or column containing the target symbol will elicit a 
P300 ERP and, by detecting this ERP, the BCI is able to identify the target row and column and thus retrieve the symbol the subject has in mind.

Ideally, performing one sequence of intensifications of each row and column would be enough to identify the target symbol. Unfortunately, the low signal-to-noise ratio of the P300 ERP makes this potential almost undetectable in single trial. The common practice is to repeat several times the sequence of intensifications, in order to average the EEG responses and increase the signal-to-noise ratio. Depending on the number of repetitions, this practice can lead to a dramatic increase of the time taken to communicate each symbol. It is thus important to work on robust and efficient feature extraction and classification techniques to reduce this number of repetitions.

An elegant way to improve the performance of a $\mathrm{BCI}$ is the detection of so-called Error-related Potentials (ErrP). ErrPs were suggested to be generated in the anterior cingulated cortex with a spatial distribution over frontocentral regions of the scalp and related to the subject's perception of an error ([13], [14]). If the first studies on the presence of an ErrP in the EEG were dealing with brain responses to errors made by the subject himself ([15], [16]), more recent work discusses the presence of such potential in the context of a BCI, when the user realizes that the interface failed to recognize properly his intention ([14], [17]-[20]). This latter definition is what we will refer to as ErrP in the article. In [18], the observation of an ErrP is obtained in the context of a vertical cursor controlled with $\mathrm{mu} /$ beta waves, while in [17] it is done in the context of a simulated BCI, where the subject manually delivers command to move a horizontal cursor. This latter experiment was then successfully reproduced where the BCI was still simulated with an a priori error rate but this time the subjects were using movement imagination ([14]). To our knowledge only researchers from the Politecnico di Milano University ([19], [20]) recently presented some work on the error potential in the context of a P300 Speller.

This paper reports on a study performed in our laboratory where 6 subjects were tested on the P300 Mind Speller ${ }^{\mathbb{R}}$ developed by our group ([21]-[23]) and for which the EEG responses to correct and incorrect feedback (moment where the BCI displays what was identified as the target symbol, see Fig.1-right) were recorded during several typing sessions. We compare our results to the studies previously mentioned and discuss the possibilities of detection of the ErrP and the ways to include it in our Mind Speller® BCI.

\section{ACQUISITION OF THE DATA}

\subsection{Material}

The EEG recordings were performed using a prototype of an ultra low-power 8-channels wireless EEG system, which consists of two parts: an amplifier coupled with a wireless transmitter and a USB stick receiver (Fig. 2a, c). The data are transmitted with a sampling frequency of $1 \mathrm{kHz}$ for each channel. The prototype was developed by the Interuniversity Microelectronics Center (IMEC, [24]). We used a brain-cap with large filling holes and sockets for active $\mathrm{Ag} / \mathrm{AgCl}$ electrodes (ActiCap, Brain Products, Fig. 2d).

The recordings were collected from eight electrodes in the frontal, central and parietal areas, namely in positions $F z$, $F C z, C z, C P z, C P 1, C P 2, P 3, P z, P 4$, according to the international 10-20 system (Fig. 2b). The reference and ground electrodes were linked to the left and right mastoids, respectively $(T P 9, T P 10)$.

The visual stimulation consisted of a matrix of 6-by- 6 symbols (Fig. 1). For both training and testing stages, each sequence of intensification consisted in the highlighting of each row and column of the matrix only once and in random order. Each highlighting lasted $100 \mathrm{~ms}$, followed by $100 \mathrm{~ms}$ of no intensification. All recordings and stimulation were performed with Matlab R2009b, the display of the stimuli and their precise timing was achieved using the Psychophysics Toolbox Extensions ([25], [26]).

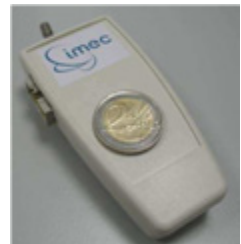

(a)

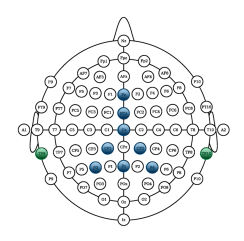

(b)

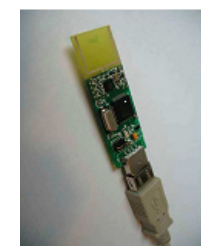

(c)

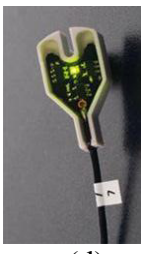

(d)
Fig. 2. (a) Wireless 8 channels amplifier. (b) Locations of the electrodes on the scalp. (c) USB stick receiver. (d) Active electrode.

\subsection{Experiment Design}

Six healthy subjects (4 male, 2 female, age 22-34, 5 right handed and 1 left handed) participated in the experiment. Each experiment lasted between $1 \mathrm{~h} 30$ and $2 \mathrm{~h} 30$, everything was done to keep the subject fully concentrated and the experiments were stopped when the participants were starting to feel tired.

The first step of the experiment was to familiarize the subjects with the Mind Speller ${ }^{\circledR}$ BCI and to train the system to recognize their P300 ERP. So, preliminary to any "mindtyping" performance, we performed a training session during which the participants were asked to focus consecutively on 8 symbols randomly selected by the interface. An indication of the symbol to focus on was first presented to the subject, then the random sequence of intensifications of all the rows and columns was repeated 10 times and finally the symbol was presented to the subject in the middle of the screen for 4 seconds (feedback moment, Fig. 1-right). This was repeated for all 8 symbols. 
Based on the data recorded during the training session, we build the classifier for the detection of the P300 ERP. The signals were beforehand filtered between 0.3 and $15 \mathrm{~Hz}$ (3rd order Butterworth filter), their mean was subtracted and they were cut into $800 \mathrm{~ms}$ epochs starting from each stimulus onset. Those epochs were then "averagedownsampled" to 80 data points (each new data point corresponds the average of the signal over its $10 \mathrm{~ms}$ surrounding window) and finally, the data of the same classes were averaged over the desired number of trials (corresponding to the desired number of repetitions of the sequence of intensification for the spelling mode).

For each trial (stimulus), we thus have 8 channels $\times 80$ data points $=640$ features to classify as a response to either a target stimulus or a non-target stimulus. A linear Support Vector Machine associated with a 10-fold cross-validation and a linesearch for the optimization of the regularization parameter was built on those training features. Training the linear SVM on 2000 data points with the modified finite Newton method proposed in [27] typically took around one minute.

The second step of the experiment was to have the subjects to use Mind Speller BCI with the previously built classifier applied online to detect the P300 ERP and identify the target symbol. They would first use the system with 10 repetitions of the sequence of intensifications, in order to make them confident about the accuracy of the system. Most of them typed their first word with almost no mistake (see last column in Table 1). As the aim was to record EEG responses to erroneous feedback, we then reduced this number of repetition to 5, 4 and even 3 according to how well the subjects were typing. Each subject spelled between 32 and 65 symbols with a number of errors comprised between 6 and 19 (see Table 1).

\section{PRESENCE OF AN ERROR POTENTIAL}

The averaged EEG responses to correct and incorrect feedback for each subject at electrode $\mathrm{FCz}$ and the grand average over subjects for each electrode are plotted in Figures 3 and 4 . We also plotted the error-minus-correct difference potentials (difference between averaged responses to erroneous feedback and averaged responses to correct feedback).

In their study, Schalk et al. ([18]) observed a errorminus-correct difference consisting of a positive potential that picked about $180 \mathrm{~ms}$ followed by a negative potential (4 subjects were tested). In [14] and [17], this difference was eliciting a first positive peak at 200ms after the feedback, followed by two larger negative and positive peaks at about $250 \mathrm{~ms}$ and $320 \mathrm{~ms}$ and a wider negative peak at $450 \mathrm{~ms}$ after the feedback (5 subjects were tested). Finally in [19] and [20] (2 and 5 subjects tested, respectively), this errorminus-correct difference showed a negative peak occurring at about $300 \mathrm{~ms}$ followed by a positive peak at around 400

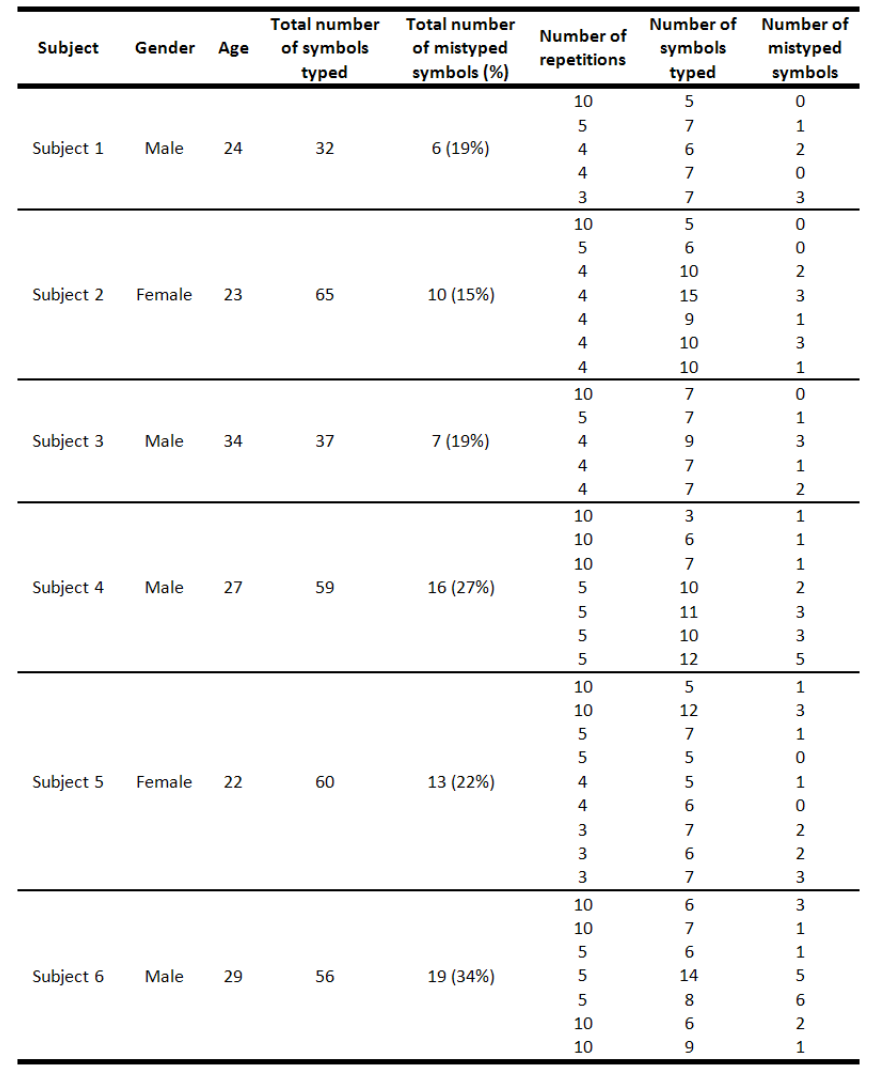

Table 1. Details of the performances of each participant. The 3 last columns detail for each session, the number of repetitions of the sequence of intensifications used to communicate each symbol, the size of the word typed by the subject and the number of wrongly typed symbols. The fourth and fifth column summarize all the typing sessions of each subject.

ms after the feedback. All three studies show quite different results concerning the shape of the ErrP. It seems thus that the shape and latency times of this potential really depends on the kind of paradigm it is associated with. In [18], the subject is trained to control a cursor with his mu/beta waves; this might already affect the shape of the ErrP. Moreover, the responses are recorded when the cursor hits the target (or the non-target) and not when the cursor moves in the intended (not-intended) direction contrarily to [14], [17] where the EEG responses to each cursor movement are recorded. Also, the latency time between feedback moments may also have an influence; if in [14], [17], the EEG feedbacks are recorded after each movement of the cursor (every 2 seconds), in [19], [20], they are recorded after each apparition of what the BCI classified as the target letter (every 15 seconds). Finally, the nature of the task itself might also influence, if a cursor moving task could involve the motor area of the brain, this is not the case of a P300 oddball paradigm.

In our case, when looking at the grand average errorminus-correct, we can observe a negative peak followed by positive one at about $320 \mathrm{~ms}$ and $450 \mathrm{~ms}$ respectively. Those potentials are the most prominent at the electrode sites $\mathrm{Fz}$ 

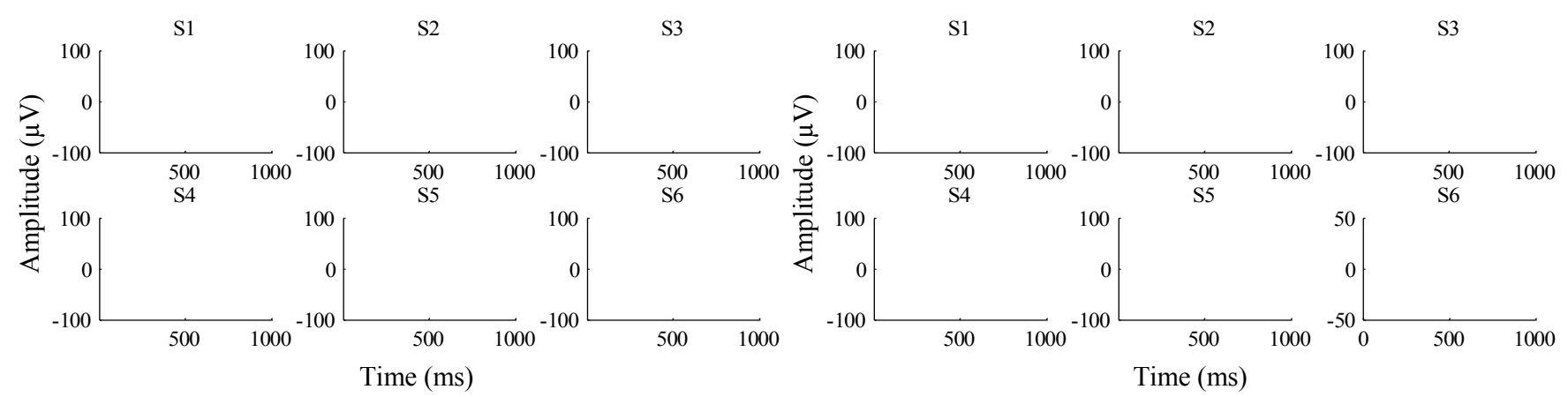

Fig. 3. EEG responses for each subject at electrode location $F C z$ for 1 second from the feedback onset. Left: EEG responses averaged over all the correct (green) and erroneous (red) feedbacks. Right: averaged error-minus-correct.
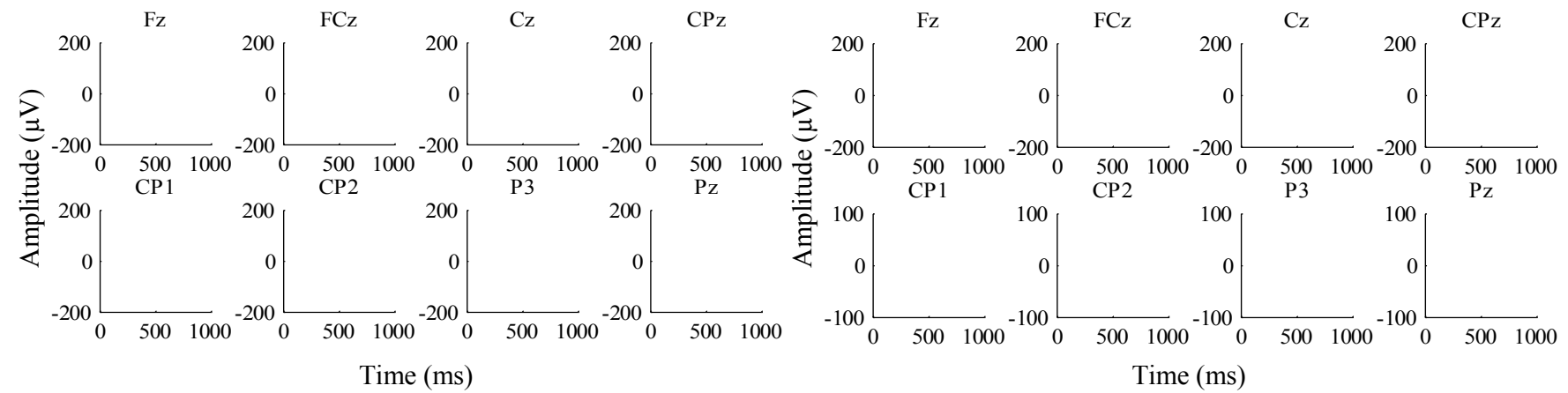

Fig. 4. EEG responses averaged over all subjects at each electrode location for 1 second from the feedback onset. Left: EEG responses averaged over all the correct (green) and erroneous (red) feedbacks. Right: averaged error-minus-correct.

and FCz. Those results are in concordance with [19], [20] where a similar P300 based speller BCI as the one presented here was used.

In order to assess the significance of the difference between responses to erroneous feedback and responses to correct feedback we analyzed the data of each subject at the electrode location FCz. We first "average-downsampled" the signals from $1000 \mathrm{~Hz}$ to $100 \mathrm{~Hz}$. Then, for each time step $i$, and for all trials of a given subject, we calculated the coefficient of determination (square of the correlation coefficient, [28]) indicating the fraction of the total variance of the EEG feedback responses $x_{k i}$, that was explained by the class $y_{k}$ of the corresponding trial $k$ (correct feedback versus erroneous feedback).

$$
\begin{aligned}
& R^{2}(i)=\frac{\operatorname{cov}\left(X_{i}, Y\right)^{2}}{\operatorname{var}\left(X_{i}\right) \operatorname{var}(Y)}, \quad i \in \llbracket 1, \text { ntimesteps } \rrbracket \\
& \text { with }\left\{\begin{array}{l}
X_{i}=\left\{x_{k i}, k \in \llbracket 1, \text { ntrials } \rrbracket, i \in \llbracket 1, \text { ntimesteps } \rrbracket\right\} \\
Y=\left\{y_{k} \in\{-1,1\}, k \in \llbracket 1, \text { ntrials } \rrbracket\right\}
\end{array}\right.
\end{aligned}
$$

Those values are plotted in Figure 5 (left). While due to the low number of trials and the low signal-to-noise ratio of the EEG signals, the values of this coefficient of determination remain quite low, we can still observe some peaks along the time. Some of those peaks have the same latency time as the negative and positive peaks that we accounted for as ErrP in the EEG feedback responses. To have an idea about how significant were those peaks with respect to the classification as ErrP and non-ErrP; we performed a permutation test at each time point (significance level 0.05, [29]). For most subject (except subject 1), the time zones corresponding to at least one of the 2 peaks associated with the ErrP were statistically significant (red diamonds on Fig. 5-left). The same study was performed, regrouping this time the data from all subjects together and the coefficient of determination for both time zones appeared statistically significant (Fig. 5-right).

If those results suggest an apparent discriminability between EEG responses to both kind of feedback, the high variability between responses of the same type among trials and subjects indicates the necessity of training the BCI to recognize the ErrP for each subject. This, in the case of the Mind Speller ${ }^{\circledR}$, can be problematic due the long time required for the acquisition of a sufficient amount of training and testing data to build and attest of the accuracy of classifier. And as shown by the comparison of the results from [14], [17]-[20], the shape of the ErrP seems closely related to the type of paradigm used for the $\mathrm{BCI}$; so that we should collect the training ErrP data in the exact context in which we want to detect them.

\section{DISCUSSION}

The first difficulty is thus to gather a sufficient amount of training data to build a classifier that would detect the ErrP. We intend to direct a study with several subjects and 

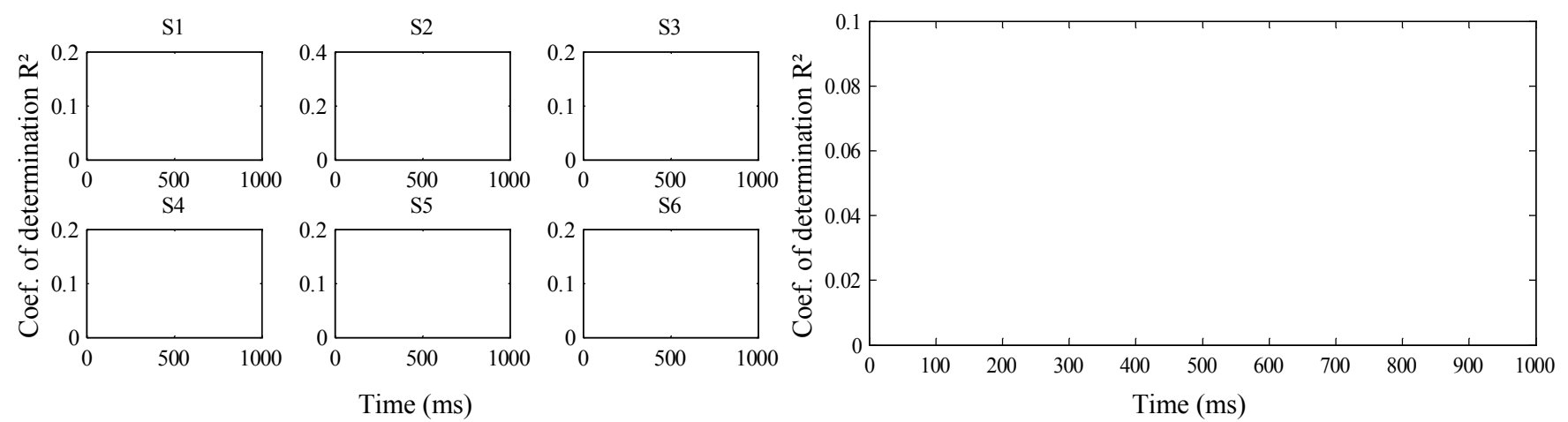

Fig. 5. Coefficient of determination versus time after feedback onset for each subject independently (left) and for all subjects together (right). The red diamonds indicates the values for which the permutation test gives a p-value lower than 0.05 .

repeated sessions to assess what would be a sufficient amount of training data. From a practical point of view performing hours of training session in order to build an ErrP classifier is not acceptable for a commercial device. A solution (also proposed in [19]) would be to use the backspace symbol as a label for the presence of an ErrP in the feedback response to the previous symbol and activate the ErrP detection once a sufficient amount of training data is reached. This would still allow the user to utilize the BCI and be familiar with the device before enhancing it with the ErrP detection tool.

The second problem is the building of the classifier; indeed contrarily to the P300 ERP, where the signals are averaged, the ErrP needs to be detected in single trial. The detection algorithm would have then to be robust enough to overcome the low signal-to-noise ratio inherent to EEG recordings and able to incrementally learn to detect an ErrP. As we want to avoid any false detection of this error potential, the classifier would also have to be strongly biased towards the minimization of the number of false positives.

Despite all these constraints, the Mind Speller ${ }^{\mathbb{B}}$ can still greatly benefit from the detection of ErrPs. Assuming that such a tool is developed, the question arises as to how this could be incorporated in our BCI. To detect the target letter, the classification algorithm computes a score for each row and column of the matrix and then selects the best row and column. From those scores we can deduce a ranking of all the symbols of the matrix. One strategy could be to simply repeat the sequence of intensifications, with eventually a lower number of repetitions, to update this ranking. But this would lead to an important increase in the time taken to communicate the symbol. Another strategy would then be, when the presence of an ErrP is detected, to select the second best letter according to the classifier's ranking. This is supported by the fact that is many cases of wrong symbol detection we could observe in our experiments that at least the column or the row of the target symbol was correctly identified. In Table 2 is presented how such a strategy could improve the performance of the Mind Speller ${ }^{\circledR}$ for our 6 subjects. We can observe a substantial reduction of the number of errors (more than 3 times less for some subjects).
If, with this strategy, not all errors are corrected, a quite important number of them are and it presents the serious advantage of minimizing the time taken for the correction. Looking at Table 2, one can see that more than half (17/33) of the remaining uncorrected symbols are ranked in the third position by our classifier. One might wonder whether it would be worth iterating this process when the symbol ranked as second appears to be wrong with the third symbol, and so on, until the correct symbol is selected. That would necessitate the correct detection of several consecutive ErrPs. Not only the theoretical probability of detection of the correct symbol will decrease proportionally to the rank of this symbol, but it is also possible that such ErrP would not be elicited several times in a row. One should check the shape of the EEG response in such a case before considering such iterative process. Nevertheless our data suggest that it would not be worth checking after the third ranked symbol.

Two more possibilities unrelated to any ErrP for improving this system can be thought of. First, one can consider weighting the scores of each symbol with an a priori probability of occurrence given the previous symbol and the typing language (e.g. Dasher, [30]). Another possibility would be the use of a dictionary to automatically correct the word once it is typed. Both ideas can perfectly be combined and offer the advantage of not increasing the time taken to communicate a word, but they would be language specific and not usable for proper nouns or non-text based communication (e.g. icon-based communication).

\section{CONCLUSION}

A first step towards the integration of ErrP detection in the P300 Mind Speller ${ }^{\circledR}$ BCI was presented. Besides the undeniable practical advantages of ErrP detection, the necessity of single trial detection, the strong noisy component of EEG signals, the high inter-subject variability and the paradigm dependency of this ERP make this task very challenging. An appropriate way to combine it with the Mind Speller ${ }^{\circledR}$ BCI has to be studied, and one should not forget about other ways of improving the system by already known techniques. 


\begin{tabular}{lccccccc}
\hline Subject & Gender & Age & $\begin{array}{c}\text { Number of } \\
\text { typing } \\
\text { sessions }\end{array}$ & $\begin{array}{c}\text { Total number } \\
\text { of symbols } \\
\text { typed }\end{array}$ & $\begin{array}{c}\text { Total number of } \\
\text { mistyped } \\
\text { symbols (\%) }\end{array}$ & $\begin{array}{c}\text { Number of mistyped symbols } \\
\text { when selecting the 2nd best } \\
\text { symbol after ErrP detection (\%) }\end{array}$ & $\begin{array}{c}\text { Ranks of the remaining } \\
\text { incorrect symbols }\end{array}$ \\
\hline Subject 1 & Male & 24 & 5 & 32 & $6(19 \%)$ & $2(6 \%)$ & 11,7 \\
Subject 2 & Female & 23 & 7 & 65 & $10(15 \%)$ & $3(5 \%)$ & $3,3,3$ \\
Subject 3 & Male & 34 & 5 & 37 & $7(19 \%)$ & $4(10 \%)$ & $4,5,3,3$ \\
Subject 4 & Male & 27 & 7 & 59 & $16(27 \%)$ & $5(12 \%)$ & $3,5,3,11,8,10,3$ \\
Subject 5 & Female & 22 & 9 & 60 & $13(22 \%)$ & $12(21 \%)$ & $8,3,3,6,4$ \\
Subject 6 & Male & 29 & 7 & 56 & $19(34 \%)$ & $4,9,6,3,3,12,3,3,5,3,3,3$ \\
\hline
\end{tabular}

Table 2. Details of the possible improvement for each subject the case of a $100 \%$ accurate ErrP detection. The $7^{\text {th }}$ column represents the amount of remaining wrong characters assuming that when an ErrP occurs, the symbol ranked in the second position by our classifier is selected as target symbol. The last column details the ranking of the remaining uncorrected symbols

\section{REFERENCES}

[1] "Is this the bionic man?" Nature, vol. 442, 2006, p. 109.

[2] P. Sajda, K. Muller, and K. Shenoy, "Brain-Computer Interfaces [from the guest editors]," IEEE Signal Processing Magazine, vol. 25, 2008, pp. 16-17.

[3] B. Pesaran, S. Musallam, and R.A. Andersen, "Cognitive neural prosthetics," Current biology: $C B$, vol. 16, 2006, pp. R77-80

[4] J.J. Vidal, "Toward direct brain-computer communication," Annual review of biophysics and bioengineering, vol. 2, 1973, pp. 157-80.

[5] P. Martinez, H. Bakardjian, and A. Cichocki, "Fully Online Multicommand Brain-Computer Interface with Visual Neurofeedback Using SSVEP Paradigm," Computational intelligence and neuroscience, vol. 2007, 2007, p. 94561.

[6] M. Cheng, X. Gao, S. Gao, and D. Xu, "Design and implementation of a brain-computer interface with high transfer rates," IEEE transactions on bio-medical engineering, vol. 49, 2002, pp. 1181-6.

[7] N. Birbaumer, A. Kübler, N. Ghanayim, T. Hinterberger, J. Perelmouter, J. Kaiser, I. Iversen, B. Kotchoubey, N. Neumann, and H. Flor, "The thought translation device (TTD) for completely paralyzed patients," IEEE transactions on rehabilitation engineering : a publication of the IEEE Engineering in Medicine and Biology Society, vol. 8, 2000, pp. 190-3.

[8] B. Blankertz, G. Dornhege, M. Krauledat, K. Müller, and G. Curio, "The non-invasive Berlin Brain-Computer Interface: fast acquisition of effective performance in untrained subjects," NeuroImage, vol. 37, 2007, pp. 539-50.

[9] J.R. Wolpaw, D.J. McFarland, and T.M. Vaughan, "Brain-computer interface research at the Wadsworth Center," IEEE Transactions on Rehabilitation Engineering, vol. 8, 2000, pp. 222-226.

[10] S.J. Luck, An Introduction to the Event-Related Potential Technique, MIT Press, 2005

[11] W.S. Pritchard, "Psychophysiology of P300," Psychological bulletin, vol. 89,1981 , pp. 506-40

[12] L.A. Farwell and E. Donchin, "Talking off the top of your head: toward a mental prosthesis utilizing event-related brain potentials," Electroencephalography and clinical neurophysiology, vol. 70, 1988, pp. 510-23.

[13] C.B. Holroyd and M.G. Coles, "The neural basis of human error processing: reinforcement learning, dopamine, and the error-related negativity," Psychological review, vol. 109, 2002, pp. 679-709.

[14] P.W. Ferrez and J. del R. Millán, "EEG-Based Brain-Computer Interaction: Improved Accuracy by Automatic Single-Trial Error Detection," Advances in Neural Information Processing Systems, vol. 20, 2007, pp. 1-8.

[15] W.J. Gehring, M.G. Coles, D.E. Meyer, and E. Donchin, "A brain potential manifestation of error-related processing," Electroencephalography and clinical neurophysiology. Supplement, vol. 44, 1995, pp. 261-72.

[16] G. Dornhege, G. Curio, and B. Blankertz, "Single Trial Detection of EEG Error Potentials: A Tool for Increasing BCI Transmission
Rates," Artificial Neural Networks - ICANN 2002, Berlin, Heidelberg: Springer Berlin Heidelberg, 2002, pp. 1137-1143.

[17] P.W. Ferrez and J. del R Millan, "Error-related EEG potentials generated during simulated brain-computer interaction," IEEE transactions on bio-medical engineering, vol. 55, 2008, pp. 923-9.

[18] G. Schalk, J.R. Wolpaw, D.J. McFarland, and G. Pfurtscheller, "EEGbased communication: presence of an error potential," Clinical neurophysiology : official journal of the International Federation of Clinical Neurophysiology, vol. 111, 2000, pp. 2138-44.

[19] B. Dal Seno, M. Matteucci, and L. Mainardi, "Online Detection of P300 and Error Potentials in a BCI Speller," Computational intelligence and neuroscience, 2010, p. 307254.

[20] G. Visconti, B. Dal Seno, M. Matteucci, and L. Mainardi, "Automatic Recognition of Error Potentials in a P300-Based BrainComputer Interface," in Proceedings of the 4th International BrainComputer Interface Workshop \& Training Course, Graz: 2008, p. 238-243.

[21] N. Chumerin, N.V. Manyakov, A. Combaz, J.A. Suykens, and M.M. Van Hulle, "An application of feature selection to on-line P300 detection in brain-computer interface," 2009 IEEE International Workshop on Machine Learning for Signal Processing, Ieee, 2009, pp. 1-6.

[22] N. Chumerin, N.V. Manyakov, A. Combaz, J.A. Suykens, R.F. Yazicioglu, T. Torfs, P. Merken, H.P. Neves, C.V. Hoof, and M.M. Van Hulle, "P300 detection based on Feature Extraction in on-line Brain-Computer Interface," KI 2009: Advances in Artificial Intelligence, 2009, pp. 339-346.

[23] A. Combaz, N.V. Manyakov, N. Chumerin, J.A. Suykens, and M.M. Van Hulle, "Feature Extraction and Classification of EEG Signals for Rapid P300 Mind Spelling," 2009 International Conference on Machine Learning and Applications, IEEE, 2009, pp. 386-391.

[24] R. Yazicioglu, P. Merken, R. Puers, and C. Van Hoof, "Low-Power Low-Noise 8-Channel EEG Front-End ASIC for Ambulatory Acquisition Systems," 2006 Proceedings of the 32nd European Solid-State Circuits Conference, IEEE, 2006, pp. 247-250.

[25] D.H. Brainard, "The Psychophysics Toolbox," Spatial Vision, vol. 10, 1997, pp. 433-436.

[26] D.G. Pelli, "The VideoToolbox software for visual psychophysics: Transforming numbers into movies," Spatial Vision, vol. 10, 1997, pp. 437-442.

[27] S. Keerthi and D. DeCoste, "A modified finite Newton method for fast solution of large scale linear SVMs," Journal of Machine Learning Research, vol. 6, 2006, pp. 341 - 361.

[28] I. Guyon and A. Elisseeff, "An introduction to variable and feature selection," The Journal of Machine Learning Research, vol. 3, 2003, p. $1157-1182$.

[29] D.S. Moore and G. P. McCabe, Introduction to the Practice of Statistics, 5th ed., W. H. Freeman, New York, 2006.

[30] D.J. Ward and D.J.C. MacKay, "Fast Hands-free Writing by Gaze Direction," Nature, vol. 418, August 2002, pp. 838. 